\title{
Fundamental Energy Requirement of Reversible Quantum Operations
}

\author{
Giulio Chiribella@, ${ }^{1,2}$ Yuxiang Yang $\odot,^{3}$ and Renato Renner ${ }^{3}$ \\ ${ }^{1}$ QICI Quantum Information and Computation Initiative, Department of Computer Science, \\ The University of Hong Kong, Pokfulam Road, Hong Kong \\ ${ }^{2}$ Department of Computer Science, University of Oxford, Parks Road, Oxford OX1 3QD, United Kingdom \\ ${ }^{3}$ Institute for Theoretical Physics, ETH Zürich, 8093 Zürich, Switzerland
}

(Received 15 November 2019; revised 14 January 2021; accepted 3 March 2021; published 15 April 2021)

\begin{abstract}
Landauer's principle asserts that any computation has an unavoidable energy cost that grows proportionally to its degree of logical irreversibility. But even a logically reversible operation, when run on a physical processor that operates on different energy levels, requires energy. Here we quantify this energy requirement, providing upper and lower bounds that coincide up to a constant factor. We derive these bounds from a general quantum resource-theoretic argument, which implies that the initial resource requirement for implementing a unitary operation within an error $\epsilon$ grows like $1 / \sqrt{\epsilon}$ times the amount of resource generated by the operation. Applying these results to quantum circuits, we find that their energy requirement can, by an appropriate design, be made independent of their time complexity.
\end{abstract}

DOI: 10.1103/PhysRevX.11.021014

Subject Areas: Quantum Physics, Quantum Information

\section{INTRODUCTION}

Landauer's tenet "information is physical" [1] is a powerful reminder that all information processing systems are necessarily subject to the laws of physics. These laws impose certain fundamental limitations. For example, the laws of quantum theory imply that perfect universally programmable quantum processors cannot exist [2]. Refinements of no-go results like this showed that they can be phrased as trade-offs between the accuracy with which the tasks can be carried out and the amount of resources available for their implementation. For example, the refinement of the above mentioned no-programming theorem asserts that the size of an approximate universally programmable quantum processor grows proportionally to $\log (1 / \epsilon)$ where $\epsilon$ quantifies the tolerated error [3-5].

Here we consider the fundamental energy requirement for implementing quantum operations. Such requirement consists of at least two different contributions, which are consequences of the second law of thermodynamics and of energy conservation in quantum mechanics, respectively. The fact that the second law of thermodynamics has implications for the energy cost of computation is known as Landauer's principle [1]. It asserts that any physical device that carries out a logically irreversible operation dissipates a certain minimum amount of energy as heat, and that this amount is proportional to the degree of

Published by the American Physical Society under the terms of the Creative Commons Attribution 4.0 International license. Further distribution of this work must maintain attribution to the author(s) and the published article's title, journal citation, and DOI. irreversibility (which may be quantified in terms of entropic quantities; see Refs. [6-9]).

In this work we are concerned with the second fundamental contribution to the energy bill. This contribution can be regarded as a consequence of energy conservation, when applied to coherent transitions across states of different energy. If a process is executed on a system with nondegenerate energy levels, then energy must be temporarily borrowed from a battery. For general quantum processes, this borrowing may occur in a superposition; i.e., the system's quantum state may consist of one branch in which energy has flown from the battery and another one in which energy has flown into the battery. To ensure that this does not lead to decoherence, the corresponding energy states of the battery must be indistinguishable. This, in turn, is possible only if the battery is large enough. Determining the corresponding energy requirement is exactly the topic of this work.

Previous approaches to quantify the energy requirement are based on the Wigner-Araki-Yanase theorem [10,11], which states that any conservation law limits the accuracy with which quantities that do not commute with the conserved quantity can be measured. The theorem implies a bound on the variance of the energy in the initial state of the battery required to implement an operation [12-17]. However, the variance does not in general provide a good bound on the size of the battery, nor on the average energy that needs to be initially stored in it. As a simple example, consider a system with $d$ equally spaced energy levels $\left\{E_{0}, \ldots, E_{d-1}\right\}$. A pure state in the superposition of energy eigenstates corresponding to $E_{0}$ and $E_{d-1}$ with amplitudes $\sqrt{1-1 / d}$ and $\sqrt{1 / d}$, respectively, has average energy (measured relative to $E_{0}=0$ ) less than $E_{1}$ and large energy variance that grows as $d$. On the other hand, even if the 
variance is fixed the energy can still take an arbitrarily large value.

Here we instead take a general resource-theoretic approach. Let $M$ be a function that quantifies the value of the different possible states of a system with respect to a resource. For example, $M(\rho)$ may be the average energy of the system when it is in state $\rho$. Furthermore, for a reversible operation $G$ on the system, we denote by $M(G)$ the maximum increase of the function $M$ when evaluated on an input state and on the corresponding output state produced by $G$. Hence, in the case where the considered resource is energy, $M(G)$ quantifies by how much the system's energy can grow when executing $G$. Assume now that we want to implement $G$ up to a precision $\epsilon$ (which we quantify in terms of the worst-case infidelity, defined below). The implementation should consist of a device that can merely carry out free operations, i.e., operations that cannot generate the resource. Such an implementation must necessarily use a battery, as illustrated in Fig. 1. Then the following general assertion can be made.

Theorem 1.-If the resource measure $M$ is monotonous, additive, and regular (see later for definitions), then every approximation of a reversible operation $G$ within error $\epsilon$ using a free device $U_{G}$ connected to a battery in state $\beta$ must satisfy

$$
M(\beta) \geq \frac{\left(M(G)+M\left(G^{\dagger}\right)\right)^{2}}{32 K_{\mathrm{S}} \sqrt{\epsilon}}-c-O(\sqrt{\epsilon}),
$$

where $c$ and $K_{\mathrm{S}}$ are constants that merely depend on $M$ and the system on which $G$ acts.

This theorem, whose proof we sketch in the Sec. II, yields in particular a lower bound on the energy requirement for implementing a reversible operation $G$.

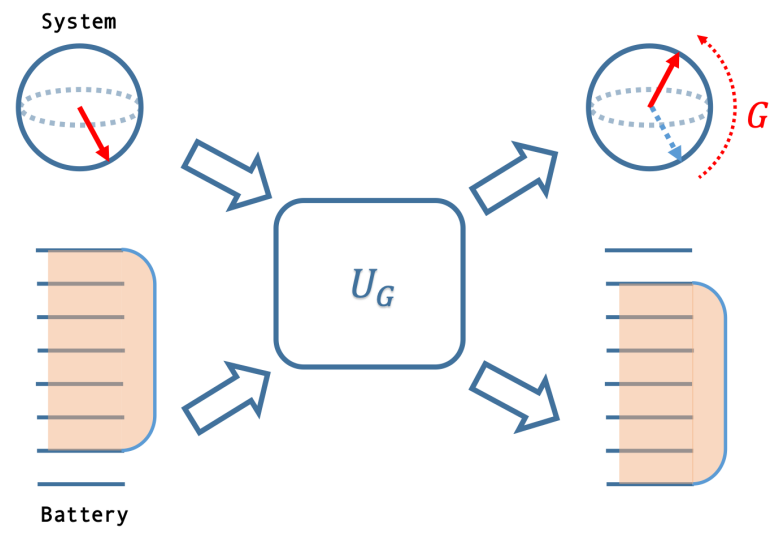

FIG. 1. Implementing a reversible gate using a battery. This figure describes a scheme that approximates a generic quantum gate $G$ that may not preserve a generic type of resource, e.g., energy, on a system. The scheme works by using a battery system that undergoes a free unitary $U_{G}$ together with the system. With the resource supplied by the battery, $G$ is approximated on the system.
Specifically, in Sec. III, we show that the average energy content $\left\langle H_{\mathrm{B}}\right\rangle$ of the battery supplying energy to the processor must be at least

$$
\left\langle H_{\mathrm{B}}\right\rangle \geq \frac{\left[\left(\lambda_{\max }-\lambda_{\min }\right)\left(\Delta_{G} H_{\mathrm{S}}\right)\right]^{2}}{32 \sqrt{\epsilon}\left\|H_{\mathrm{S}}\right\|}-O(\sqrt{\epsilon}),
$$

where $\left\|H_{\mathrm{S}}\right\|$ is the operator norm of the system's Hamiltonian, $\lambda_{\max }\left(\lambda_{\min }\right)$ denotes the maximal (minimal) eigenvalue, and $\Delta_{G} H_{\mathrm{S}}=G^{\dagger} H_{\mathrm{S}} G-H_{\mathrm{S}}$ is the change of the system's Hamiltonian induced by the action of the gate $G$. We have assumed, without loss of generality, that the minimum energy is zero for both the system and the battery, and thus $\left\|H_{\mathrm{S}}\right\|$ is equal to the maximum energy of the system. The bound (1) states that the average energy of the battery should be above the ground state energy by an amount determined by the energy change operator $\Delta_{G} H_{\mathrm{S}}$, the system's energy scale $\left\|H_{\mathrm{S}}\right\|$, and the error $\epsilon$.

While the bound (1) depends on the particular operation $G$, by maximizing over all such reversible operations we obtain a bound on the energy requirement of a universal quantum processor operating on a system $\mathrm{S}$ with a given Hamiltonian $H_{\mathrm{S}}$,

$$
\left\langle H_{\mathrm{B}}\right\rangle \geq \frac{\left\|H_{\mathrm{S}}\right\|}{8 \sqrt{\epsilon}}-O(\sqrt{\epsilon}) .
$$

This bound is tight up to a constant factor. More precisely, assuming that the system has equally spaced energy levels, we show by an explicit construction, described in Sec. IV, that

$$
\left\langle H_{\mathrm{B}}\right\rangle \leq \frac{\pi\left\|H_{\mathrm{S}}\right\|}{2 \sqrt{\epsilon}} .
$$

Taking together these two bounds, we have thus established that the fundamental energy requirement for operating on $\mathrm{S}$ grows as $\left\|H_{\mathrm{S}}\right\| / \sqrt{\epsilon}$. Note that if the system's Hamiltonian is fully degenerate, i.e., $\left\|H_{\mathrm{S}}\right\|=0$, then energy conservation does not imply an energy requirement. Besides the average energy, we show that the energy spread of the battery is lower bounded by $\left\|H_{\mathrm{S}}\right\| / \sqrt{\epsilon}$, and the tightness of the bound can again be achieved with the construction that led to Eq. (3).

Finally, we determine how the energy requirement of a quantum circuit depends on its complexity. Previous works considered implementations of quantum circuits where each gate is powered by an independent battery $[12,14]$ [see Fig. 2(a)]. The energy requirement then obviously grows linearly with the number of nonconservative gates, making complex computations energetically demanding. In contrast, we show that the energy requirement of quantum circuits is independent of their complexity. For this we consider an implementation that uses a single battery to power all gates in the circuit [see Fig. 2(b)]. It turns out that 


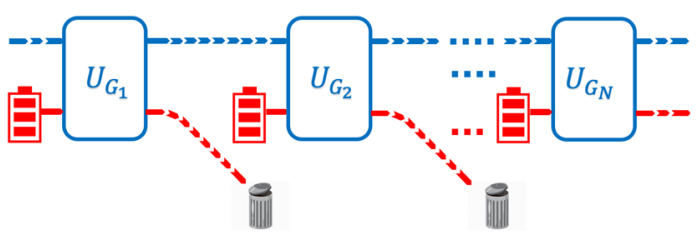

(a)

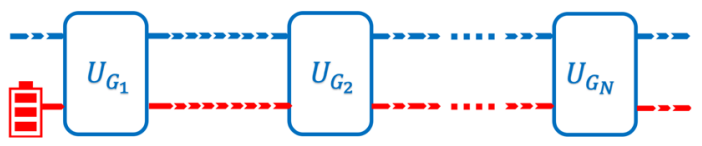

(b)

FIG. 2. Multiple and single battery implementations of quantum computation. Two different setups of energy-preserving quantum computation are compared. Panel (a) depicts the multiple battery implementation that has often been considered in previous work [12-16,19], where each single gate of the circuit is equipped with an individual battery that is discarded after the gate is implemented. In contrast, in this work we consider the single battery implementation as illustrated in (b), where a single battery provides energy for the whole circuit and is reused after the implementation of each individual gate.

energy can be recycled from one gate to the next, and that the energy requirement for a sequence of gates is exactly equal to the energy requirement of the overall gate resulting from their composition. Hence, the energy requirement depends only on the size of the computational register, but not on the time complexity of the computation. For quantum computations with classical inputs and outputs, such as Shor's algorithm, we further show that our implementation is exact and the energy requirement is just the energy needed to write down the output of the computation. This may be regarded as the quantum analog of a classical result by Fredkin and Toffoli [18], who studied the fundamental energy constraints that the classical laws of physics impose on computation.

\section{LOWER BOUND ON GENERAL RESOURCE REQUIREMENT}

In this section, we spell out the assumptions underlying Theorem 1 and describe the main proof idea. (The full proof is provided in the Appendix A.) For this we take a resource-theoretic viewpoint; i.e., we start from a given set of free operations that is closed under composition [20]. Let $U_{G}$ be such a free operation that acts on both a system $\mathrm{S}$ and a battery $\mathrm{B}$, which is initialized in state $\beta$. The resulting operation on $S$ is then described by the quantum channel,

$$
\mathcal{E}_{G}(\cdot)=\operatorname{Tr}_{\mathrm{B}}\left[U_{G}(\cdot \otimes \beta) U_{G}^{\dagger}\right]
$$

where $\beta$ is the initial state of the battery and $\operatorname{Tr}_{\mathrm{B}}$ denotes the partial trace over the battery's Hilbert space.
To quantify how well the operation $\mathcal{E}_{G}$ approximates a desired gate $G$, we use the worst-case fidelity $F_{\text {wc }}$ between the output of $G$ and $\mathcal{E}_{G}$ for any input, which may also be correlated to an external reference system $\mathrm{R}$. That is, explicitly,

$F_{\mathrm{wc}}:=\inf _{\mathrm{R}} \inf _{|\Psi\rangle \in \mathcal{H}_{\mathrm{S}} \otimes \mathcal{H}_{\mathrm{R}}} \operatorname{Tr}\left[\left(\mathcal{E}_{G} \otimes \mathcal{I}_{\mathrm{R}}\right)(\Psi)\left(\mathcal{G} \otimes \mathcal{I}_{\mathrm{R}}\right)(\Psi)\right]$,

with $\Psi:=|\Psi\rangle\langle\Psi|, \mathcal{G}(\cdot)=G \cdot G^{\dagger}$, and $\mathcal{I}_{\mathrm{R}}$ denoting the identity map on $L\left(\mathcal{H}_{\mathrm{R}}\right)$, the space of linear operators on $\mathcal{H}_{\mathrm{R}}$. We say that an implementation has error $\epsilon$ if $F_{\mathrm{wc}}=1-\epsilon$. The use of this error measure is justified by the fact that the resource requirements, in the case of energy as discussed in the Introduction, can be bounded tightly in terms of $\epsilon$ (up to a constant). We also note that the fidelity is easy to evaluate and widely used to quantify the quality of gates in quantum computation. Moreover, it may be related to other measures of distance, e.g., the diamond norm [21] (see Ref. [22] for a definition) via the inequalities $1-\sqrt{F_{\mathrm{wc}}} \leq \frac{1}{2}\left\|\mathcal{E}_{G}-\mathcal{G}\right\|_{\diamond} \leq \sqrt{1-F_{\mathrm{wc}}}$. In Appendix D, we show that the dependence of the energy requirement on the diamond norm also scales as $1 / \sqrt{\epsilon}$, up to a factor that may however depend on the system's dimension.

Theorem 1 is a general resource-theoretic statement, which merely depends on general properties of the measure $M$ used to quantify resourcefulness. Specifically, for any given system, $M$ is a function of the density operator of that system such that the following holds.

(1) Monotonicity. $M$ is nonincreasing under free operations and partial trace.

(2) Additivity on product states. $M(\rho \otimes \sigma)=$ $M(\rho)+M(\sigma)$.

(3) Regularity. There exists a constant $c \in \mathbb{R}$ and, for any system $\mathrm{S}$, a Lipschitz constant $K_{\mathrm{S}} \geq 0$, such that $|M(\rho)-M(\sigma)| \leq K_{\mathrm{S}}\|\rho-\sigma\|_{1}+c$ for any states $\rho$ and $\sigma$ of system $\mathrm{S}$, and such that $K_{\mathrm{S}}$ is subadditive; i.e., $K_{A B} \leq K_{A}+K_{B}$ for any systems $A$ and $B$.

With these definitions in place, we can now proceed to the proof of Theorem 1. Let $\mathcal{V}_{G}(\cdot):=\mathcal{U}_{G}(\cdot \otimes \beta)$ with $\mathcal{U}_{G}(\cdot)=U_{G} \cdot U_{G}^{\dagger}$ be the evolution defined in Eq. (4), but before tracing out the battery $\mathrm{B}$. Using techniques from Refs. [23-25], we show (see Appendix A) that the channel $\mathcal{V}_{G}$ is close to $\mathcal{G} \otimes \beta^{\prime}$, where $\beta^{\prime}$ is a suitable battery state [26]. Because of its additivity property, it is useful to measure this closeness in terms of the diamond norm $\|\cdot\|_{\diamond}[22]$ :

$$
\left\|\mathcal{V}_{G}-\mathcal{G} \otimes \beta^{\prime}\right\|_{\diamond} \leq 2 \sqrt{\epsilon}
$$

But this means that approximately there is no entanglement between the system and the battery after the evolution, and the battery ends up in a state close to $\beta^{\prime}$. Conversely, the state $\beta^{\prime}$ may be used to approximately implement the inverse gate $G^{\dagger}$, using the gate $U_{G}^{\dagger}$, i.e., 


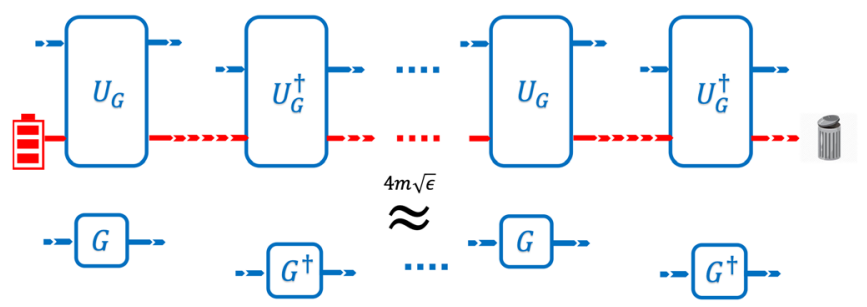

FIG. 3. Approximating $m$ uses of a gate and its inverse. If a unitary gate $G$ can be implemented with small error, then the battery can be reused $2 m$ times, approximately implementing $m$ uses of the gates $G$ and $G^{\dagger}$. As a consequence, the state of the battery should be able to provide $m$ times the maximum resource generation of $G$ and $G^{\dagger}$ up to a correction.

$$
\left\|\mathcal{G}^{-1} \otimes \beta-\mathcal{V}_{G}^{\prime}\right\|_{\diamond} \leq 2 \sqrt{\epsilon}
$$

with $\mathcal{V}_{G}^{\prime}(\cdot):=\mathcal{U}_{G}^{-1}\left(\cdot \otimes \beta^{\prime}\right)$.

According to Eqs. (6) and (7) we may thus implement the gate $G^{\dagger}$ after gate $G$, thereby returning the ancilla approximately to its initial state. Repeating this procedure $m$ (for any $m \in \mathbb{N}$ ) times, i.e., composing $m$ implementations of $G$ and of $G^{\dagger}$ in alternating order as illustrated in Fig. 3, we still approximate each of them within an error bounded by $4 m \sqrt{\epsilon}$. Note that the circuit in the lower half of the figure increases the resource value $M$ by virtue of $G$ and $G^{\dagger}$. To approximate this increase of resource, the circuit in the upper half of the figure must use the battery, because all the other operations in the circuit are free and therefore resource nongenerating. Hence, the amount of resource generated by $m$ uses of $G$ and $G^{\dagger}$, i.e., $m$ times $M(G)+M\left(G^{\dagger}\right)$, must be matched by the battery. Evaluating this amount leads to the bound stated in Theorem 1 .

\section{LOWER BOUND ON ENERGY REQUIREMENT}

To obtain Eq. (1) we apply Theorem 1 to the resource theory where the free operations are energy-preserving channels [27,28]. For any system, the resource function $M$ is defined by $M(\rho)=\operatorname{Tr}[H \rho]$, where $H$ is the system's Hamiltonian (with the minimum energy set to zero) and $\rho$ is the system's density operator. This resource function is additive on product states and it is nonincreasing under energy-preserving channels and partial trace. Moreover, the inequality $|\operatorname{Tr}(\rho-\sigma) H| \leq\|\rho-\sigma\|_{1} \cdot\|H\|$ shows that the function $M$ is Lipschitz continuous with Lipschitz constant $K_{\mathrm{S}}=\|H\|$, equal to the energy scale of the system under consideration, and $c=0$. Finally, we note that $M(G)=\max _{|\psi\rangle \in \mathcal{H}_{\mathrm{S}}}\left\langle\psi\left|G^{\dagger} H_{\mathrm{S}} G\right| \psi\right\rangle-\left\langle\psi\left|H_{\mathrm{S}}\right| \psi\right\rangle=$ $\lambda_{\max }\left(\Delta_{G} H_{\mathrm{S}}\right) \quad$ and $\quad M\left(G^{\dagger}\right)=\max _{|\psi\rangle \in \mathcal{H}_{\mathrm{S}}}\left\langle\psi\left|G H_{\mathrm{S}} G^{\dagger}\right| \psi\right\rangle-$ $\left\langle\psi\left|H_{\mathrm{S}}\right| \psi\right\rangle=-\lambda_{\text {min }}\left(\Delta_{G} H_{\mathrm{S}}\right)$, where $\lambda_{\max }\left(\lambda_{\min }\right)$ denotes the maximal (minimal) eigenvalue. Inserting all this into Theorem 1, we immediately obtain the desired bound (1) on the average energy content $\left\langle H_{\mathrm{B}}\right\rangle=$ $\operatorname{Tr}\left[H_{\mathrm{B}} \beta\right]$ of the battery B required to implement $G$.

In a similar way we can also derive a lower bound on the required total capacity $\left\|H_{\mathrm{B}}\right\|$ of the battery. For this we first apply Theorem 1 to the resource function $M^{\prime}(\rho):=$ $\operatorname{Tr} \rho(\|H\| \cdot I-H)$ to obtain the bound,

$$
\left\|H_{\mathrm{B}}\right\|-\left\langle H_{\mathrm{B}}\right\rangle \geq \frac{\left[\left(\lambda_{\max }-\lambda_{\min }\right)\left(\Delta_{G} H_{\mathrm{S}}\right)\right]^{2}}{32 \sqrt{\epsilon}\left\|H_{\mathrm{S}}\right\|}-O(\sqrt{\epsilon}) .
$$

Taking the worst case $G$, which satisfies $\lambda_{\max }\left(\Delta_{G} H_{\mathrm{S}}\right)=$ $-\lambda_{\min }\left(\Delta_{G} H_{\mathrm{S}}\right)=\left\|H_{\mathrm{S}}\right\|$, and combining this with bound (1), we find a bound on the maximum energy (or the capacity) of the battery,

$$
\left\|H_{\mathrm{B}}\right\| \geq \frac{\left\|H_{\mathrm{S}}\right\|}{4 \sqrt{\epsilon}}-O(\sqrt{\epsilon})
$$

This and Eq. (2) are lower bounds on the energy requirement of a universal processor, able to implement arbitrary gates on system $\mathrm{S}$ with error $\epsilon$ or less. While Eq. (2) quantifies the energy requirement in terms of the average energy that a battery must contain, Eq. (9) refers to the battery's total capacity.

Theorem 1 also provides bounds on other types of resources, such as coherence [29-32]. A concrete example is the relative entropy of coherence [29] $C(\rho):=$ $S\left(\rho_{\text {diag }}\right)-S(\rho)$, with $S$ denoting the von Neumann entropy and $\rho_{\text {diag }}$ the diagonal part of $\rho$ in the energy eigenbasis. Here the theorem yields the bound (see Appendix B),

$$
C(\beta) \geq \frac{\left(C(G)+C\left(G^{\dagger}\right)\right)^{2}}{32 \sqrt{\epsilon} \log d_{\mathrm{S}}}-O(1),
$$

on the initial coherence that the battery must provide, where $d_{\mathrm{S}}$ is the dimension of the system on which $G$ acts, and $C(G)$ is the amount of coherence generated by the gate $G$. For gates like the generalized Hadamard gate, this quantity can be as large as $\log d_{\mathrm{S}}$. Therefore, the minimum amount of coherence required to operate a universal quantum processor scales like $\log d_{\mathrm{S}} / \sqrt{\epsilon}$.

We have shown that the requirement for energy and coherence both follow an $1 / \sqrt{\epsilon}$ scaling. The same scaling also characterizes the standard deviation of the energy, as observed in previous works [12-17].

\section{ATTAINING THE BOUND}

We now show that the bound (2) can be attained with a suitable choice of battery state and interaction between the system and the battery. In this section, we assume that the system has equally spaced energy levels, which is the case, for example, if it consists of $n$ identical individual qubits. We denote the spacing by $\hbar \omega$. 
The implementation uses a battery with equally spaced energy levels with spacing $\hbar \omega$, ranging from 0 to $\left\|H_{\mathrm{B}}\right\|=$ $R\left\|H_{\mathrm{S}}\right\|$, where $R$ is an integer, assumed to be larger than 2 for later convenience. At the beginning, the battery is initialized in a superposition of energy eigenstates with sine-shaped amplitudes [33],

$|\beta\rangle=\sqrt{\frac{2}{L}} \sum_{E_{\mathrm{B}}=\left\|H_{\mathrm{S}}\right\|}^{(R-1)\left\|H_{\mathrm{S}}\right\|} \sin \left(\frac{\left(E_{\mathrm{B}}-\left\|H_{\mathrm{S}}\right\|+\hbar \omega\right) \pi}{\hbar \omega L}\right)\left|E_{\mathrm{B}}\right\rangle$,

where the summation runs in steps of $\hbar \omega$, and $L=$ $(R-2)\left\|H_{\mathrm{S}}\right\| /(\hbar \omega)+2$. Note that the lowest and highest energy levels are unoccupied. This allows the battery to both supply and absorb energy from the system.

For the interaction between the system and the battery we adopt a construction from Refs. [34-36], suitably adapted to unitary gates on finite-dimensional systems. Denote by $E_{\mathrm{S}, x}$ the energy of $\left|\psi_{x}\right\rangle$. For a given value $E$ of the total energy, and for every $x$ satisfying the condition

$$
E-\left\|H_{\mathrm{B}}\right\| \leq E_{\mathrm{S}, x} \leq E,
$$

we define the eigenstates,

$$
|x, E\rangle:=\left|\psi_{x}\right\rangle \otimes\left|E-E_{\mathrm{S}, x}\right\rangle .
$$

Then, we denote by $E_{\text {ok }}$ the set of values of the total energy such that condition (12) is satisfied for every $x=1, \ldots, d_{\mathrm{S}}$, or equivalently, the set of values $E$ satisfying the condition $\left\|H_{\mathrm{S}}\right\| \leq E \leq\left\|H_{\mathrm{B}}\right\|$. For every $E \in \mathrm{E}_{\mathrm{ok}}$, define the partial isometry,

$$
U_{G}^{(E)}:=\sum_{x, y=0}^{d_{S}-1}\left\langle\psi_{x}|G| \psi_{y}\right\rangle|x, E\rangle\langle y, E|,
$$

which acts as the unitary gate $G$ in the eigenspace with total energy $E$. To make the computation reversible on the whole system $\mathrm{SB}$, we set $U_{G}$ to be the unitary gate,

$$
U_{G}:=\sum_{E \in \mathrm{E}_{\mathrm{ok}}} U_{G}^{(E)}+\sum_{E \notin \mathrm{E}_{\mathrm{ok}}} P_{E},
$$

where $P_{E}$ is the projector on the subspace with total energy $E$.

In Appendix C, we show that the worst-case fidelity of the above implementation is lower bounded as

$$
F_{\mathrm{wc}} \geq 1-\left(\frac{\pi\left(\lambda_{\max }-\lambda_{\min }\right)\left(\Delta_{G} H_{\mathrm{S}}\right)}{4\left\langle H_{\mathrm{B}}\right\rangle}\right)^{2}\left[1+O\left(\frac{\left\|H_{\mathrm{S}}\right\|}{\left\langle H_{\mathrm{B}}\right\rangle}\right)\right],
$$

and therefore the energy requirement is upper bounded as

$$
\left\langle H_{\mathrm{B}}\right\rangle \leq \frac{\pi\left(\lambda_{\max }-\lambda_{\min }\right)\left(\Delta_{G} H_{\mathrm{S}}\right)}{4 \sqrt{\epsilon}}\left[1+O\left(\frac{\left\|H_{\mathrm{S}}\right\|}{\left\langle H_{\mathrm{B}}\right\rangle}\right)\right] .
$$

In the worst case over all possible gates, one has $\left(\lambda_{\max }-\lambda_{\text {min }}\right)\left(\Delta_{G} H_{\mathrm{S}}\right)=2\left\|H_{\mathrm{S}}\right\|$, matching the lower bound (2) up to a constant factor of $4 \pi$.

The error $\epsilon$ depends on the parameter $R$ that characterizes the battery state (11). Observing that the energy of the sine state is $\left\langle H_{\mathrm{B}}\right\rangle=R\left\|H_{\mathrm{S}}\right\| / 2$, we obtain the dependency $R \approx \pi\left(\lambda_{\text {max }}-\lambda_{\text {min }}\right)\left(\Delta_{G} H_{\mathrm{S}}\right) /\left(2 \sqrt{\epsilon}\left\|H_{\mathrm{S}}\right\|\right)$. Therefore, the battery capacity of this implementation is $\left\|H_{\mathrm{B}}\right\|=$ $R\left\|H_{\mathrm{S}}\right\| \approx \pi\left(\lambda_{\max }-\lambda_{\min }\right)\left(\Delta_{G} H_{\mathrm{S}}\right) /(2 \sqrt{\epsilon})$. Taking the worst case $G$, the capacity of the battery is approximately

$$
\left\|H_{\mathrm{B}}\right\| \approx \frac{\pi\left\|H_{\mathrm{S}}\right\|}{\sqrt{\epsilon}}
$$

matching the lower bound (9) up to a constant of $4 \pi$.

\section{ENERGY-EFFICIENT QUANTUM COMPUTATION}

We established the minimum energy requirement of one single quantum operation. But what about a computation that consists of many individual steps? One way to implement the computation is to assign an individual battery to each gate and to replace the gate by its conservative approximation. However, this approach leads to a heavy energy toll. If each gate is powered by an individual battery of energy $\left\langle H_{\mathrm{B}}\right\rangle$, then bound (2) implies that the error cannot decrease faster than $1 /\left\langle H_{\mathrm{B}}\right\rangle^{2}$. The error (infidelity) is a lower bound on the trace distance, which in the worst case increases linearly with the number of gates. The linear increase implies that at most $O\left(\left\langle H_{\mathrm{B}}\right\rangle^{2}\right)$ gates can be combined together with tolerable error. For a circuit of $N$ nonconservative gates, this means that the energy of each individual battery should grow at least as $\sqrt{N}$, with a total energy requirement scaling at least as $N^{3 / 2}$. In other words, the energy requirement depends on the number of nonconservative gates, just as in traditional models of dissipative computation.

We now show that, in fact, quantum computation can be implemented with an amount of energy that is independent of the circuit depth. To do so, we propose a scheme of computation where energy is recycled from one computational step to the next. The computation is performed on $n$ identical qubits, each with Hamiltonian $H_{\mathrm{S}}^{(1)}$ and energy gap $\left\|H_{\mathrm{S}}^{(1)}\right\|=\hbar \omega$, and uses a single battery of capacity $C_{\mathrm{B}}=R n\left\|H_{\mathrm{S}}^{(1)}\right\|$, where $R$ is an integer depending on the desired level of accuracy. For an elementary gate $G$ acting on a subset of $k$ qubits, we let the battery and the $k$ qubits interact through the energy-preserving gate $U_{G}$ in Eq. (15). The energy subspaces on which the gate $U_{G}$ acts nontrivially correspond to the energy values $\mathrm{E}_{\mathrm{ok}}^{(k)}=$ $\left\{E \mid k\left\|H_{\mathrm{S}}^{(1)}\right\| \leq E \leq\left\|H_{\mathrm{B}}\right\|\right\}$. Now, consider the total energy of the $n$ qubits and the battery. For every two gates $G_{1}$ and $G_{2}$, one has the property, 


$$
U_{G_{1}} U_{G_{2}} P_{\mathrm{ok}}^{(n)}=U_{G_{1} G_{2}} P_{\mathrm{ok}}^{(n)},
$$

where $P_{\mathrm{ok}}^{(n)}$ is the projector on the eigenspaces of the total energy in $\mathrm{E}_{\mathrm{ok}}^{(n)}$. The above relation means that the local interactions of the battery with subsets of qubits are enough to generate every global interaction between the battery and all the qubits involved in the computation. Hence, the computation can be realized by preparing the battery in the state (11), with $\left\|H_{\mathrm{S}}\right\|=n\left\|H_{\mathrm{S}}^{(1)}\right\|$. For a computation consisting of $N$ gates $\left(G_{i}\right)_{i=1}^{N}$, the energy requirement does not depend on $N$, but only on the unitary $G=G_{N} \cdots G_{2} G_{1}$ that describes the overall computation. Since the gate $G$ acts on at most $n$ qubits, the energy requirement for implementing any computation with accuracy $\epsilon$ is at most $\pi n \hbar \omega /(2 \sqrt{\epsilon})$.

It is worth noting that, if the computation is only required to work on a subset of input states, the energy requirement can be lower. For example, suppose that a computation has classical input and classical output, as in Shor's algorithm and in many other quantum algorithms. In this case, every computation can be implemented exactly by setting the battery in the initial state with energy $n \hbar \omega$, and then using the interaction (15) for every gate (see Appendix E).

\section{CONCLUSIONS}

We derived a bound on the resources that are required to approximately implement a reversible quantum operation. We found that, for a general class of resources, which include energy as a special case, the resource requirement grows as $1 / \sqrt{\epsilon}$, where $\epsilon$ is the approximation error (Theorem 1 ). Furthermore, in the case where the resource is energy, the bound is attainable within a constant factor, provided that the target system has equally spaced energy levels. A typical example for such a situation is a quantum processor acting on $n$ identical qubits. For a computation, this minimum energy requirement is, remarkably, achievable even if the computation is carried out by a complex quantum circuit with many individual unitary gates. In this case, we showed that the battery state can be recycled from one computational step to the next, making the energy requirement independent of how the computation is decomposed.

Our bound on the energy requirement is unrelated to the second law of thermodynamics: it follows from the conservation of energy, and it is present even if the evolution is entirely reversible. Nonetheless, our energy requirement can be compared quantitatively with the thermodynamical work requirement associated to Landauer's principle, which is present when the evolution is irreversible. Landauer's principle sets the work cost of erasing information from a single qubit to $K_{B} T$, where $K_{B}$ is Boltzmann's constant and $T$ is the system's temperature. For superconducting qubits, assuming an operation temperature of the order of $1 \mathrm{~K}$, the Landauer cost is of the order of $10^{-23} \mathrm{~J}$. Our bounds (2) and (3), on the other hand, introduce a new energy requirement that depends on the Hamiltonian of the qubit system and the desired implementation accuracy. For transmon superconducting qubits, the energy gap between $|0\rangle$ and $|1\rangle$ is around the order of $10 \mathrm{GHz}$ [37,38], implying an energy requirement of $\sim 10^{-24} \times \epsilon^{-1 / 2} \mathrm{~J}$. The energy requirement is thus comparable to the energy cost predicted by Landauer's principle.

Like Landauer's principle, our results must be understood as fundamental limitations imposed by the laws of physics. At least for today's few-qubit devices, which require large cooling and control machinery external to the actual quantum processors, the fundamental energy requirement as given by Eqs. (2) and (3) merely represents a minor part of the overall energy consumption. However, as quantum technology is being developed further, the energy required, e.g., for cooling, will most likely scale less than linearly with the number of qubits, and its contribution to the overall energy bill thus becomes less dominant. Analogously to how the fundamental bounds of classical thermodynamics have helped us optimizing engines, a theory of the thermodynamics of computation can guide the optimization of computations with respect to their energy consumption. The bounds presented here may be regarded as a contribution to such a theory.

\section{ACKNOWLEDGMENTS}

This work is supported by the National Natural Science Foundation of China through Grant No. 11675136, the Hong Kong Research Grant Council through Grants No. 17326616 and No. 17300918, the Foundational Questions Institute through Grant No. FQXi-RFP3-1325, the Croucher Foundation, the Swiss National Science Foundation via the National Center for Competence in Research "QSIT" as well as via Projects No. 200020_165843 and No. 200021_188541, and the ETH Pauli Center for Theoretical Studies. We thank Daniel Ebler for designing the figures.

\section{APPENDIX A: PROOF OF THEOREM 1}

We assume that the resource function $M$ satisfies monotonicity and regularity (properties 1 and 3 in the main text). In addition, additivity (Property 2 in the main text) can be relaxed to

(2) Subadditivity on product states. $M(\rho \otimes \sigma) \leq$ $M(\rho)+M(\sigma)$.

Under these properties, we prove a more general result on the resource requirement, which reduces to Theorem 1 (which we prove as Corollary 3) when property 2 is substituted by additivity.

Theorem 2.-Every approximation of the gate $G$ within error $\epsilon$ using a free gate $U_{G}$ and a battery in the state $\beta$ must satisfy the inequality,

$$
M(\beta) \geq m \bar{M}_{m}\left(G \otimes G^{\dagger}\right)-8 \sqrt{\epsilon} K_{\mathrm{S}} m^{2}-c,
$$


for every $m \in \mathbb{N}^{*}$, where $\bar{M}_{m}(U)$ is the regularized resource generation $[39,40]$ of $m$ uses of a quantum gate $U$ acting on a system $\mathrm{S}$. Explicitly, $\bar{M}_{m}(U)$ is defined as

$$
\bar{M}_{m}(U):=\max _{\rho_{m}} \frac{1}{m}\left\{\left(M\left(\mathcal{U}^{\otimes m}\left(\rho_{m}\right)\right)-M\left(\rho_{m}\right)\right),\right.
$$

where $\mathcal{U}(\cdot):=U(\cdot) U^{\dagger}$ and the maximum is taken over all $m$-partite states.

We remark that Eq. (A1) is the general formula that can be used to further derive resource inequalities with simpler forms: $\bar{M}_{m}$ can scale differently, e. g., $\bar{M}_{m}=O(1)$ or $\bar{M}_{m}=O(m)$, for different resource theories. One can then optimize over all $m \in \mathbb{N}^{*}$ to get the scaling of the resource requirement with respect to the error, which depends on the resource theory under consideration.

The proof of Theorem 2 is based on the following lemma, in which we use the notation $F_{\mathrm{wc}}(\mathcal{C}, \mathcal{D}):=$ $\inf _{\mathrm{R}} \inf _{|\Psi\rangle \in \mathcal{H}_{\mathrm{S}} \otimes \mathcal{H}_{\mathrm{R}}} F\left[\left(\mathcal{C} \otimes \mathcal{I}_{\mathrm{R}}\right)(|\Psi\rangle\langle\Psi|),\left(\mathcal{D} \otimes \mathcal{I}_{\mathrm{R}}\right)(|\Psi\rangle\langle\Psi|)\right]$.

Lemma 1.-Let $G$ be a gate acting on system $\mathrm{S}, U_{G}$ a gate acting on system $\mathrm{SB}, \beta$ be a state of system $\mathrm{B}$, let $\mathcal{V}_{G}$ be the channel from $\mathrm{S}$ to $\mathrm{SB}$ defined by $\mathcal{V}_{G}(\rho):=$ $U_{G}(\rho \otimes \beta) U_{G}^{\dagger}$, and let $\mathcal{E}_{G}$ be the channel from $\mathrm{S}$ to $\mathrm{S}$ defined by $\mathcal{E}_{G}(\rho):=\operatorname{Tr}_{\mathrm{B}}\left[\mathcal{V}_{G}(\rho)\right]$. Then, there exists a state $\beta^{\prime}$ of system $\mathrm{B}$, such that $\left\|\mathcal{V}_{G}-\mathcal{G} \otimes \beta^{\prime}\right\|_{\diamond} \leq$ $2 \sqrt{1-F\left(\mathcal{E}_{G}, \mathcal{G}\right)}$.

Proof.-Let $\tilde{\beta}$ be a purification of $\beta$ with purifying system E. Then, the channel $\tilde{\mathcal{V}}_{G}(\cdot):=\left(\mathcal{U}_{G} \otimes \mathcal{I}_{\mathrm{E}}\right)(\cdot \otimes \tilde{\beta})$ is a Stinespring dilation of the channel $\mathcal{E}_{G}$ [41].

The Uhlmann's theorem for gates [23,42] guarantees that there exists a Stinespring dilation of the gate $G$, say $G \otimes \tilde{\beta}^{\prime}$ for some pure state $\tilde{\beta}^{\prime}$, such that the fidelity between $\tilde{\mathcal{V}}_{G}$ and $G \otimes \tilde{\beta}^{\prime}$ is equal to the fidelity between $\mathcal{E}_{G}$ and $G$, namely,

$$
F_{\mathrm{wc}}\left(\mathcal{G} \otimes \tilde{\beta}^{\prime}, \tilde{\mathcal{V}}_{G}\right)=F_{\mathrm{wc}}\left(\mathcal{G}, \mathcal{E}_{G}\right)
$$

Tracing out E, we obtain

$$
F_{\mathrm{wc}}\left(\mathcal{G} \otimes \beta^{\prime}, \mathcal{V}_{G}\right) \geq F_{\mathrm{wc}}\left(\mathcal{G}, \mathcal{E}_{G}\right)
$$

where $\mathcal{V}_{G}(\cdot):=\mathcal{U}_{G}(\cdot \otimes \beta)$. Since $\mathcal{V}_{G}$ and $\mathcal{G} \otimes \beta^{\prime}$ are extensions of the original channels, the converse inequality also holds, namely, $F_{\text {wc }}\left(\mathcal{G} \otimes \beta^{\prime}, \mathcal{V}_{G}\right) \geq F_{\text {wc }}\left(\mathcal{G}, \mathcal{E}_{G}\right)$. Hence, the inequality is in fact an equality.

Then, the Fuchs-Van de Graph inequality [43] yields the relation,

$$
\left\|\mathcal{V}_{G}-\mathcal{G} \otimes \beta^{\prime}\right\|_{\diamond} \leq 2 \sqrt{1-F_{\mathrm{wc}}\left(\mathcal{G}, \mathcal{E}_{G}\right)} .
$$

Corollary 1.-Let $\mathcal{V}_{G}^{\prime}$ be the channel from $\mathrm{S}$ to SB defined by $\mathcal{V}_{G}^{\prime}(\rho):=U_{G}^{\dagger}\left(\rho \otimes \beta^{\prime}\right) U_{G}$. Then, one has $\left\|\mathcal{V}_{G}^{\prime}-\mathcal{G}^{\dagger} \otimes \beta\right\|_{\diamond} \leq 2 \sqrt{1-F\left(\mathcal{E}_{G}, \mathcal{G}\right)}$.
Proof.-The inequality follows from the unitary invariance of the diamond norm:

$$
\begin{aligned}
\left\|\mathcal{V}_{G}^{\prime}-\mathcal{G}^{\dagger} \otimes \beta\right\|_{\diamond} & =\left\|\mathcal{U}_{G} \circ \mathcal{V}_{G}^{\prime} \circ \mathcal{G}-\mathcal{U}_{G} \circ\left(\mathcal{G}^{\dagger} \otimes \beta\right) \circ \mathcal{G}\right\|_{\diamond} \\
& =\left\|\mathcal{G} \otimes \beta-\mathcal{V}_{G}\right\|_{\diamond} \\
& \leq 2 \sqrt{1-F_{\mathrm{wc}}\left(\mathcal{G}, \mathcal{E}_{G}\right)}
\end{aligned}
$$

Corollary 2.-Let $\mathcal{C}_{G}$ be the multipartite channel corresponding to the circuit in Fig. 3 of the main text. Then, one has the bound $\left\|\mathcal{C}_{G}-\left(\mathcal{G} \otimes \mathcal{G}^{-1}\right)^{\otimes m} \otimes \beta\right\|_{\diamond} \leq$ $4 m \sqrt{1-F_{\mathrm{wc}}\left(\mathcal{G}, \mathcal{E}_{G}\right)}$.

Proof--It follows from the unitary invariance and triangle inequality of the diamond norm, combined with the bounds in Lemma 1 and Corollary 1.

Proof of Theorem 2.-Consider the input state $\rho_{\text {in }}:=$ $\rho_{\text {in }}^{(2 m)} \otimes \beta$, where $\rho_{\text {in }}^{(2 m)}$ is an arbitrary input state on $2 m$ identical copies of the system. Let $\rho_{\text {out }}$ be the output state resulting from the approximate circuit in Fig. 3 of the main text. Using the monotonicity of the function $M$, we obtain the relation,

$$
M\left(\operatorname{Tr}_{\mathrm{B}}\left[\rho_{\text {out }}\right]\right) \leq M\left(\rho_{\text {out }}\right) \leq M\left(\rho_{\text {in }}\right) .
$$

By property 2 (subadditivity), we have $M\left(\rho_{\text {in }}\right) \leq$ $M(\beta)+M\left(\rho_{\text {in }}^{(2 m)}\right)$. Then, we have the bound,

$$
M(\beta) \geq M\left(\operatorname{Tr}_{\mathrm{B}}\left[\rho_{\text {out }}\right]\right)-M\left(\rho_{\text {in }}^{(2 m)}\right) .
$$

Now, we apply property 3 (regularity) to the ideal output and to its approximation, whose difference has trace norm at most $4 m \sqrt{\epsilon}$ from the actual output state $\rho_{\text {out }}$, due to Corollary 2. Noticing that the Lipschitz constant for the system $(\mathrm{S} \otimes \mathrm{S})^{\otimes m}$ is upper bounded by $2 m K_{\mathrm{S}}$, the bound (A8) becomes

$$
\begin{aligned}
M(\beta) \geq & M\left(\left(\mathcal{G} \otimes \mathcal{G}^{-1}\right)^{\otimes m}\left(\rho_{\mathrm{in}}^{(2 m)}\right)\right)-M\left(\rho_{\mathrm{in}}^{(2 m)}\right) \\
& -8 \sqrt{\epsilon} K_{\mathrm{S}} m^{2}-c
\end{aligned}
$$

which holds for any $m \in \mathbb{N}^{*}$ and for any input state $\rho_{\mathrm{in}}^{(2 m)}$. Maximizing over all inputs fixing $m$, we have

$$
M(\beta) \geq m \bar{M}_{m}\left(G \otimes G^{\dagger}\right)-8 \sqrt{\epsilon} K_{\mathrm{S}} m^{2}-c,
$$

where $\bar{M}_{m}(U)$ is defined by Eq. (A2).

When $M$ is additive on product states, i.e., $M(\rho \otimes \sigma)=M(\rho)+M(\sigma)$, the general bound (A1) can be simplified by finding an $m$-independent lower bound on $\bar{M}_{m}$.

Corollary 3 (Theorem 1 in the main text).-When $M$ is additive, the resource requirement in the battery becomes 


$$
M(\beta) \geq \frac{\left(M(G)+M\left(G^{\dagger}\right)\right)^{2}}{32 K_{\mathrm{S}} \sqrt{\epsilon}}-c-2 K_{\mathrm{S}} \sqrt{\epsilon},
$$

where $M(G):=\max _{\rho} M\left(G \rho G^{\dagger}\right)-M(\rho)$ is the amount of resource generated by the gate $G$.

For additive $M$ the function $\bar{M}_{m}$ is monotonically increasing with $m$. Since $M(G)=\bar{M}_{1}(G)$, it is obviously upper bounded by $\bar{M}_{m}(G)$.

Proof.-Let us consider a product form input $\rho_{\text {in }}^{(2 m)}=$ $(\rho \otimes \sigma)^{\otimes m}$ to the circuit. Since $M$ satisfies additivity, we have

$$
\begin{aligned}
\bar{M}_{m}\left(G \otimes G^{\dagger}\right) \\
\quad \geq \frac{1}{m}\left(M\left(\left(\mathcal{G}(\rho) \otimes \mathcal{G}^{-1}(\sigma)\right)^{\otimes m}\right)-M\left((\rho \otimes \sigma)^{\otimes m}\right)\right) \\
\quad=(M(\mathcal{G}(\rho))-M(\rho))+\left(M\left(\mathcal{G}^{-1}(\sigma)\right)-M(\sigma)\right),
\end{aligned}
$$

for every $m \in \mathbb{N}^{*}$. Choosing $\rho$ and $\sigma$ to be the maximal resource generating inputs for $\mathcal{G}$ and $\mathcal{G}^{-1}$, respectively, we have

$$
\bar{M}_{m}\left(G \otimes G^{\dagger}\right) \geq M(G)+M\left(G^{\dagger}\right) .
$$

Substituting into Eq. (A1), we get

$$
M(\beta) \geq m\left(M(G)+M\left(G^{\dagger}\right)\right)-8 \sqrt{\epsilon} K_{\mathrm{S}} m^{2}-c .
$$

Finally, we obtain the lower bound (A10) on the amount of resource in the battery by maximizing the bound over all possible $m \in \mathbb{N}$. The optimal choice $m^{*} \in \mathbb{N}$ satisfies $\left|m^{*}-\left(M(G)+M\left(G^{\dagger}\right)\right) / 16 \sqrt{\epsilon} K_{\mathrm{S}}\right| \leq 1 / 2$. Substituting into Eq. (A13) we get (A10).

We conclude by mentioning a further extension of Theorem 2 that takes into account the possibility of applying the gate $G$ on part of a composite system.

Corollary 4.-Every approximation of the gate $G$ within error $\epsilon$ using a free gate $U_{G}$ and a battery in the state $\beta$ must satisfy the inequality,

$$
M(\beta) \geq m \bar{M}_{m}\left(G \otimes I_{\mathrm{R}} \otimes G^{\dagger} \otimes I_{\mathrm{R}}\right)-8 \sqrt{\epsilon} K_{\mathrm{SR}} m^{2}-c,
$$

for every $m \in \mathbb{N}^{*}$, where $\mathrm{R}$ is a reference system.

Proof.-The result follows from the application of Theorem 2 to the gate $G \otimes I_{\mathrm{R}}$, observing that, by definition, the diamond norm and the worst-case fidelity are invariant under addition of a reference system.

\section{APPENDIX B: APPLICATION TO THE RESOURCE THEORY OF COHERENCE}

The resource of quantum coherence [29-31,44-46] can be characterized operationally in terms of different sets of free operations, such as strictly incoherent operations [44], maximally incoherent operations $[47,48]$, dephasing covariant operations [31,45,46], phase covariant operations [31], and physically incoherent operations $[45,46]$. These operations are defined relative to a fixed basis $\{|i\rangle\}$, and preserve the set of incoherent states, of the form $\rho=\sum_{i} p_{i}|i\rangle\langle i|$. For composite systems, it is understood that the fixed basis of the composite system is the product of the fixed bases for the components.

For the purpose of our bound, the choice of the set of free operations is not critical. As a measure of resource, we consider the relative entropy of coherence [29],

$$
C(\rho):=S\left(\rho_{\text {diag }}\right)-S(\rho),
$$

$S$ denoting the von Neumann entropy of quantum states and $\rho_{\text {diag }}$ being the diagonal part of $\rho$ in the energy basis. This measure of coherence satisfies the properties 1 (monotonicity) and 2 (additivity on product states). It also satisfies property 3 , as shown by the following.

Proposition 1.-The function $C: L\left(\mathbb{C}^{d}\right) \rightarrow \mathbb{R}, C(\rho)=$ $S\left(\rho_{\text {diag }}\right)-S(\rho)$ satisfies the inequality $|\mathcal{C}(\rho)-\mathcal{C}(\sigma)| \leq$ $\log d\|\rho-\sigma\|_{1}+2$.

Proof--For any two states $\rho$ and $\sigma$ in a $d$-dimensional Hilbert space, the difference of their entropies is bounded by the Fannes-Audenaert inequality $[49,50]$,

$|S(\rho)-S(\sigma)| \leq \frac{\log d}{2}\|\rho-\sigma\|_{1}+h_{2}\left(\|\rho-\sigma\|_{1} / 2\right)$,

where $h_{2}(p):=-p \log p-(1-p) \log (1-p)$ is the binary entropy, upper bounded by one for any $p$. For our purpose, it is enough to use the relaxed version of the above inequality:

$$
|S(\rho)-S(\sigma)| \leq \frac{\log d}{2}\|\rho-\sigma\|_{1}+1 .
$$

Now, let us consider the difference of the relative entropies of coherence (B1) between $\rho$ and $\sigma$. We have

$$
|C(\rho)-C(\sigma)| \leq|S(\rho)-S(\sigma)|+\left|S\left(\rho_{\text {diag }}\right)-S\left(\sigma_{\text {diag }}\right)\right| .
$$

Applying Eq. (B3) to both terms on the right-hand side of the above inequality and noticing that $\left\|\rho_{\text {diag }}-\sigma_{\text {diag }}\right\|_{1} \leq$ $\|\rho-\sigma\|_{1}$ (monotonicity of trace distance under data processing), we have

$$
|C(\rho)-C(\sigma)| \leq \log d\|\rho-\sigma\|_{1}+2 .
$$

Therefore, we have $K=\log d$ and $c=2$.

Using the above proposition and Theorem 1 of the main text, we obtain a lower bound on the initial coherence in the battery:

$$
C(\beta) \geq \frac{\left(C(G)+C\left(G^{\dagger}\right)\right)^{2}}{32 \sqrt{\epsilon} \log d_{\mathrm{S}}}-2
$$


Gates like the generalized Hadamard gate have coherence generation up to $\log d_{\mathrm{S}}$. Therefore, the minimum amount of required coherence in a quantum processor is lower bounded as

$$
C(|\beta\rangle\langle\beta|) \geq \frac{\log d_{\mathrm{S}}}{8 \sqrt{\epsilon}}-2 .
$$

\section{APPENDIX C: LOWER BOUND ON THE ACCURACY}

In the following we determine the lower bound (16) on $F_{\mathrm{wc}}$. Notice that $F_{\mathrm{wc}}$ can be rewritten as $F_{\mathrm{wc}}=$ $\inf _{\mathrm{R}} \inf _{|\Psi\rangle \in \mathcal{H}_{S} \otimes \mathcal{H}_{\mathrm{R}}} F_{\Psi}$, where

$$
F_{\Psi}:=\operatorname{Tr}\left[\left(\mathcal{E}_{G} \otimes \mathcal{I}_{\mathrm{R}}\right)(\Psi)\left(\mathcal{G} \otimes \mathcal{I}_{\mathrm{R}}\right)(\Psi)\right] .
$$

To evaluate the this fidelity, we observe that the gate $U_{G}$, defined in Eq. (15), can be expressed as $U_{G}=U_{G}^{(\mathrm{ok})}+P_{\perp}^{(\mathrm{ok})}$, where $P_{\perp}^{(\mathrm{ok})}$ is the projector on the eigenstates of the total energy outside the set $\mathrm{E}_{\mathrm{ok}}$, and $U_{G}^{(\mathrm{ok})}$ is the partial isometry,

$$
\begin{aligned}
U_{G}^{(\mathrm{ok})} & :=\sum_{x, y} G_{x y}\left|\psi_{x}\right\rangle\left\langle\psi_{y}\right| \otimes S^{(x y)}, \\
S^{(x y)} & :=\sum_{E \in \mathrm{E}_{\mathrm{ok}}}\left|E-E_{\mathrm{S}, x}\right\rangle\left\langle E-E_{\mathrm{S}, y}\right|,
\end{aligned}
$$

where we use the shorthand $A_{x y}=\left\langle\psi_{x}|A| \psi_{y}\right\rangle$ for a generic operator $A \in L\left(\mathcal{H}_{\mathrm{S}}\right)$. Observe that the battery state (11) is defined so that the joint state of the system and the battery has full support in energy subspaces with $E \in \mathrm{E}_{\mathrm{ok}}$. Substituting Eq. (C2) into Eq. (C1), one has the expression,

$$
F_{\Psi}=\sum_{x, y, z, t=0}^{d_{S}-1} C_{x y z t}\left(\rho G^{\dagger}\right)_{x y} G_{y x} G_{z t}^{\dagger}(G \rho)_{t z},
$$

where $\rho$ is the marginal state $\rho=\operatorname{Tr}_{\mathrm{R}}[|\Psi\rangle\langle\Psi|]$ and $C_{x y z t}=\left\langle\beta\left|S_{z t}^{\dagger} S_{x y}\right| \beta\right\rangle$.

The quantity $C_{x y z t}$ can be explicitly evaluated as

$$
\begin{aligned}
C_{x y z t}= & \sum_{k=\left\|H_{\mathrm{S}}\right\|+y}^{(R-1)\left\|H_{\mathrm{S}}\right\|-x} \frac{2}{L} \sin \left(\frac{\left(k-z+t-2\left\|H_{\mathrm{S}}\right\|+1\right) \pi}{L}\right) \\
& \times \sin \left(\frac{\left(k+x-y-2\left\|H_{\mathrm{S}}\right\|+1\right) \pi}{L}\right) \\
= & \frac{(L-x-y-1) \cos \left(\frac{(x-y-z+t) \pi}{L}\right)}{L} \\
& +\frac{\sin \left(\frac{(x+y+1) \pi}{L}\right) \cos \left(\frac{\left(2\left\|H_{\mathrm{S}}\right\|-t+z\right) \pi}{L}\right)}{L \sin \left(\frac{\pi}{L}\right)} \\
= & 1-\frac{(x-y-z+t)^{2} \pi^{2}}{8\left\langle H_{\mathrm{B}}\right\rangle^{2}}\left[1+O\left(\frac{\left\|H_{\mathrm{S}}\right\|}{\left\langle H_{\mathrm{B}}\right\rangle}\right)\right],
\end{aligned}
$$

where the last step follows from the definition of $L$. Inserting the above expression into Eq. (C4) and rearranging the different terms, we obtain

$$
F_{\Psi}=1-\frac{\pi^{2} \operatorname{Var}\left(\Delta_{G} H_{\mathrm{S}}\right)}{4\left\langle H_{\mathrm{B}}\right\rangle^{2}}\left[1+O\left(\frac{\left\|H_{\mathrm{S}}\right\|}{\left\langle H_{\mathrm{B}}\right\rangle}\right)\right],
$$

where $\operatorname{Var}\left(\Delta_{G} H_{\mathrm{S}}\right)$ denotes the variance of the operator $\Delta_{G} H_{\mathrm{S}}$ on the state $|\Psi\rangle$. Noting that $\operatorname{Var}\left(\Delta_{G} H_{\mathrm{S}}\right) \leq$ $\left(\left(\lambda_{\max }-\lambda_{\min }\right)\left(\Delta_{G} H_{\mathrm{S}}\right) / 2\right)^{2}$, Eq. (C6) implies the following bound on the worst-case fidelity:

$F_{\mathrm{wc}} \geq 1-\left(\frac{\pi\left(\lambda_{\max }-\lambda_{\min }\right)\left(\Delta_{G} H_{\mathrm{S}}\right)}{4\left\langle H_{\mathrm{B}}\right\rangle}\right)^{2}\left[1+O\left(\frac{\left\|H_{\mathrm{S}}\right\|}{\left\langle H_{\mathrm{B}}\right\rangle}\right)\right]$.

\section{APPENDIX D: ENERGY REQUIREMENT IN TERMS OF THE DIAMOND NORM ERROR}

Here we show that the energy requirement still scales as $1 / \sqrt{\epsilon}$, when the error $\epsilon$ is measured by the diamond norm error [22] instead of $1-F_{\mathrm{wc}}$.

On one hand, since the diamond norm error upper bounds the worst-case infidelity via the inequality $1-\sqrt{F_{\mathrm{wc}}} \leq \frac{1}{2}\left\|\mathcal{E}_{G}-\mathcal{G}\right\|_{\diamond}$, we have $1-F_{\mathrm{wc}} \leq 2 \epsilon$ when the diamond norm error is at most $\epsilon$. The proof of the lower bound [cf. Eq. (2)] goes through and we get

$$
\left\langle H_{\mathrm{B}}\right\rangle \geq \frac{\left\|H_{\mathrm{S}}\right\|}{8 \sqrt{2 \epsilon}}-O(\sqrt{\epsilon}) .
$$

On the other hand, the output state of the construction (15) can be expressed as

$$
\begin{aligned}
\mathcal{E}_{G} & \otimes \mathcal{I}_{\mathrm{R}}(\Psi) \\
& =\sum_{x, y, z, t=0}^{d_{\mathrm{S}}-1} C_{x y z t} G_{x y}\left|\psi_{x}\right\rangle\left\langle\psi_{y}|\Psi| \psi_{t}\right\rangle\left\langle\psi_{z}\right| G_{z t}^{*},
\end{aligned}
$$

where $C_{x y z t}$ is given by Eq. (C5). The diamond norm error can be obtained by taking the worst case over $\Psi$ of the following quantity:

$\frac{1}{2} \| \sum_{x, y, z, t=0}^{d_{S}-1}\left(C_{x y z t}-1\right) G_{x y}\left|\psi_{x}\right\rangle\left\langle\psi_{y}|\Psi| \psi_{t}\right\rangle\left\langle\psi_{z}\right| G_{z t}^{*} \|_{1}$.

The quantity $1-C_{x y z t}$ can be upper bounded as

$$
1-C_{x y z t} \leq \frac{\pi^{2}\left(d_{\mathrm{S}}-1\right)^{2}}{2\left\langle H_{\mathrm{B}}\right\rangle^{2}}\left[1+O\left(\frac{\left\|H_{\mathrm{S}}\right\|}{\left\langle H_{\mathrm{B}}\right\rangle}\right)\right] \forall x, y, z, t,
$$

since $x, y, z, t \in\left\{0, \ldots, d_{\mathrm{S}}-1\right\}$. Substituting into Eq. (D3), the diamond norm error $\epsilon$ can be upper bounded as 


$$
\begin{aligned}
\epsilon & \leq \frac{\pi^{2}\left(d_{\mathrm{S}}-1\right)^{2}}{2\left\langle H_{\mathrm{B}}\right\rangle^{2}}\left[1+O\left(\frac{\left\|H_{\mathrm{S}}\right\|}{\left\langle H_{\mathrm{B}}\right\rangle}\right)\right] \max _{\Psi}\left\|G \Psi G^{\dagger}\right\|_{1} \\
& =\frac{\pi^{2}\left(d_{\mathrm{S}}-1\right)^{2}}{2\left\langle H_{\mathrm{B}}\right\rangle^{2}}\left[1+O\left(\frac{\left\|H_{\mathrm{S}}\right\|}{\left\langle H_{\mathrm{B}}\right\rangle}\right)\right] .
\end{aligned}
$$

Finally, we have

$$
\left\langle H_{\mathrm{B}}\right\rangle \leq \frac{\pi\left(d_{\mathrm{S}}-1\right)}{\sqrt{2 \epsilon}}\left[1+O\left(\frac{\left\|H_{\mathrm{S}}\right\|}{\left\langle H_{\mathrm{B}}\right\rangle}\right)\right] .
$$

In summary, we derived both upper and lower bounds on $\left\langle H_{\mathrm{B}}\right\rangle$ in terms of the diamond norm error. Since the two bounds have matching scaling, we conclude that the energy requirement scales as $1 / \sqrt{\epsilon}$, independently of whether one measures the error in terms of the worst-case infidelity or in terms of the diamond norm error.

\section{APPENDIX E: PERFECT IMPLEMENTATION OF QUANTUM COMPUTATION}

Here we consider a generic quantum algorithm that starts by preparing an energy eigenstate state $\left|\psi_{x}\right\rangle$ and ends by measuring the energy eigenbasis. The overall action of the algorithm can be described by a unitary gate $G$. We observe that the input-output relation induced by gate $G$ can be reproduced without errors using the interaction (15). For an initial state $\left|\psi_{x}\right\rangle$ of the system, one prepares the battery in the state $\left|E-E_{\mathrm{S}, x}\right\rangle$, so that the joint state is

$$
|x, E\rangle=\left|\psi_{x}\right\rangle \otimes\left|E-E_{\mathrm{S}, x}\right\rangle,
$$

where the total energy $E \in \mathrm{E}_{\mathrm{ok}}$. Then the initial state of the system and the battery can be expressed as $|x, E\rangle$. The effect of the interaction (15) can be expressed as $U_{G}|x, E\rangle=\sum_{y} g_{y, x}|y, E\rangle$, where $g_{x, y}=\left\langle\psi_{y}|G| \psi_{x}\right\rangle$ is the matrix element of $G$. The system ends up in the state $\sum_{y}\left|g_{y, x}\right|^{2}\left|\psi_{y}\right\rangle\left\langle\psi_{y}\right|$. Therefore, when measuring in the energy eigenbasis in the end, the probability of getting the outcome $y$ is exactly $\left|g_{y, x}\right|^{2}$, which is the same as for the original algorithm $G$.

[1] R. Landauer, Irreversibility and Heat Generation in the Computing Process, IBM J. Res. Dev. 5, 183 (1961).

[2] M. A. Nielsen and I. Chuang, Quantum Computation and Quantum Information (Cambridge University Press, Cambridge, England, 2000).

[3] D. Pérez-García, Optimality of Programmable Quantum Measurements, Phys. Rev. A 73, 052315 (2006).

[4] A. M. Kubicki, C. Palazuelos, and D. Pérez-García, Resource Quantification for the No-Programing Theorem, Phys. Rev. Lett. 122, 080505 (2019).

[5] Y. Yang, R. Renner, and G. Chiribella, Optimal Universal Programming of Unitary Gates, Phys. Rev. Lett. 125, 210501 (2020).
[6] T. Sagawa and M. Ueda, Minimal Energy Cost for Thermodynamic Information Processing: Measurement and Information Erasure, Phys. Rev. Lett. 102, 250602 (2009).

[7] D. Reeb and M. M. Wolf, An Improved Landauer Principle with Finite-Size Corrections, New J. Phys. 16, 103011 (2014).

[8] P. Faist, F. Dupuis, J. Oppenheim, and R. Renner, The Minimal Work Cost of Information Processing, Nat. Commun. 6, 7669 (2015).

[9] P. Faist and R. Renner, Fundamental Work Cost of Quantum Processes, Phys. Rev. X 8, 021011 (2018).

[10] E. Wigner, Die Messung Quantenmechanischer Operatoren, Z. Phys. A 133, 101 (1952).

[11] H. Araki and M. M. Yanase, Measurement of Quantum Mechanical Operators, Phys. Rev. 120, 622 (1960).

[12] M. Ozawa, Conservative Quantum Computing, Phys. Rev. Lett. 89, 057902 (2002).

[13] M. Ozawa, Uncertainty Principle for Quantum Instruments and Computing, Int. J. Quantum. Inform. 01, 569 (2003).

[14] J. Gea-Banacloche and M. Ozawa, Minimum-Energy Pulses for Quantum Logic Cannot be Shared, Phys. Rev. A 74, 060301(R) (2006).

[15] T. Karasawa and M. Ozawa, Conservation-Law-Induced Quantum Limits for Physical Realizations of the Quantum NOT Gate, Phys. Rev. A 75, 032324 (2007).

[16] T. Karasawa, J. Gea-Banacloche, and M. Ozawa, Gate Fidelity of Arbitrary Single-Qubit Gates Constrained by Conservation Laws, J. Phys. A 42, 225303 (2009).

[17] H. Tajima, N. Shiraishi, and K. Saito, Uncertainty Relations in Implementation of Unitary Operations, Phys. Rev. Lett. 121, 110403 (2018).

[18] E. Fredkin and T. Toffoli, Conservative Logic, Int. J. Theor. Phys. 21, 219 (1982).

[19] J. Ikonen, J. Salmilehto, and M. Möttönen, Energy-Efficient Quantum Computing, npj Quantum Inf. 3, 17 (2017).

[20] B. Coecke, T. Fritz, and R. W. Spekkens, A Mathematical Theory of Resources, Inf. Comput. 250, 59 (2016).

[21] A. Y. Kitaev, Quantum Computations: Algorithms and Error Correction, Russ. Math. Surv. 52, 1191 (1997).

[22] The diamond norm is defined as the maximum trace distance between the outputs of the two channels, maximized over all input states and over all possible reference systems [21].

[23] D. Kretschmann, D. Schlingemann, and R. F. Werner, The Information-Disturbance Tradeoff and the Continuity of StineSpring's Representation, IEEE Trans. Inf. Theory 54, 1708 (2008).

[24] G. Chiribella, G. M. D’Ariano, P. Perinotti, D. Schlingemann, and R. Werner, A Short Impossibility Proof of Quantum Bit Commitment, Phys. Lett. A 377, 1076 (2013).

[25] G. Gutoski, A. Rosmanis, and J. Sikora, Fidelity of Quantum Strategies with Applications to Cryptography, Quantum 2, 89 (2018).

[26] Similar techniques have been used in Refs. [17,51].

[27] G. Chiribella and Y. Yang, Optimal Quantum Operations at Zero Energy Cost, Phys. Rev. A 96, 022327 (2017).

[28] C. Sparaciari, J. Oppenheim, and T. Fritz, Resource Theory for Work and Heat, Phys. Rev. A 96, 052112 (2017).

[29] T. Baumgratz, M. Cramer, and M. B. Plenio, Quantifying Coherence, Phys. Rev. Lett. 113, 140401 (2014). 
[30] A. Winter and D. Yang, Operational Resource Theory of Coherence, Phys. Rev. Lett. 116, 120404 (2016).

[31] I. Marvian and R.W. Spekkens, How to Quantify Coherence: Distinguishing Speakable and Unspeakable Notions, Phys. Rev. A 94, 052324 (2016).

[32] A. Streltsov, G. Adesso, and M. B. Plenio, Colloquium: Quantum Coherence as a Resource, Rev. Mod. Phys. 89, 041003 (2017).

[33] V. Bužek, R. Derka, and S. Massar, Optimal Quantum Clocks, Phys. Rev. Lett. 82, 2207 (1999).

[34] P. Skrzypczyk, A. J. Short, and S. Popescu, Extracting Work from Quantum Systems, arXiv:1302.2811.

[35] J. Åberg, Catalytic Coherence, Phys. Rev. Lett. 113, 150402 (2014).

[36] M. Navascués and S. Popescu, How Energy Conservation Limits Our Measurements, Phys. Rev. Lett. 112, 140502 (2014).

[37] J. Koch, T. M. Yu, J. Gambetta, A. A. Houck, D. I. Schuster, J. Majer, A. Blais, M. H. Devoret, S. M. Girvin, and R. J. Schoelkopf, Charge-Insensitive Qubit Design Derived from the Cooper Pair Box, Phys. Rev. A 76, 042319 (2007).

[38] M. Kjaergaard, M. E. Schwartz, J. Braumüller, P. Krantz, J. I.-J. Wang, S. Gustavsson, and W. D. Oliver, Superconducting Qubits: Current State of Play, Annu. Rev. Condens. Matter Phys. 11, 369 (2020).

[39] Z.-W. Liu and A. Winter, Resource Theories of Quantum Channels and the Universal Role of Resource Erasure, arXiv:1904.04201.

[40] Y. Liu and X. Yuan, Operational Resource Theory of Quantum Channels, Phys. Rev. Research 2, 012035 (2020).
[41] V. Paulsen, Completely Bounded Maps and Operator Algebras (Cambridge University Press, Cambridge, England, 2002), Vol. 78.

[42] A. Uhlmann, The "Transition Probability" in the State

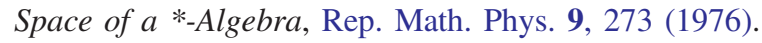

[43] C. A. Fuchs and J. Van de Graaf, Cryptographic Distinguishability Measures for Quantum-Mechanical States, IEEE Trans. Inf. Theory 45, 1216 (1999).

[44] B. Yadin, J. Ma, D. Girolami, M. Gu, and V. Vedral, Quantum Processes Which Do Not Use Coherence, Phys. Rev. X 6, 041028 (2016).

[45] E. Chitambar and G. Gour, Critical Examination of Incoherent Operations and a Physically Consistent Resource Theory of Quantum Coherence, Phys. Rev. Lett. 117, 030401 (2016).

[46] E. Chitambar and G. Gour, Comparison of Incoherent Operations and Measures of Coherence, Phys. Rev. A 94, 052336 (2016).

[47] J. Aberg, Quantifying Superposition, arXiv:quant-ph/ 0612146.

[48] M. G. Díaz, K. Fang, X. Wang, M. Rosati, M. Skotiniotis, J. Calsamiglia, and A. Winter, Using and Reusing Coherence to Realize Quantum Processes, Quantum 2, 100 (2018).

[49] M. Fannes, A Continuity Property of the Entropy Density for Spin Lattice Systems, Commun. Math. Phys. 31, 291 (1973).

[50] K. M. Audenaert, A Sharp Continuity Estimate for the von Neumann Entropy, J. Phys. A 40, 8127 (2007).

[51] D. Kretschmann, D. W. Kribs, and R. W. Spekkens, Complementarity of Private and Correctable Subsystems in Quantum Cryptography and Error Correction, Phys. Rev. A 78, 032330 (2008). 